\title{
FORMULATION, OPTIMIZATION, AND CHARACTERIZATION OF BIOCOMPATIBLE INHALABLE D-CYCLOSERINE-LOADED ALGINATE-CHITOSAN NANOPARTICLES FOR PULMONARY DRUG DELIVERY
}

\author{
JESSY SHAJI*, MAHMOOD SHAIKH
}

Department of Pharmaceutics, Prin. K. M. Kundnani College of Pharmacy, Mumbai, Maharastra, India. Email: jessy.shaji@gmail.com Received: 21 March 2016, Revised and Accepted: 13 July 2016

\section{ABSTRACT}

Objective: To prepare Nanoparticulate dosage form having improved drug bioavailability and reduced dosing frequency of antitubercular drugs which will helps in improving patient compliance in the treatment of multi-drug resistant tuberculosis (MDR-TB).

Methods: Ionotropic gelation method was used to prepare D-cycloserine (D-CS)-loaded alginate-chitosan nanoparticles, and the particles are characterized by their particle size and morphology using particle size analyzer and scanning electron microscopy (SEM). X-ray diffraction (XRD), differential scanning calorimetry (DSC), and Fourier-transformed infrared (FTIR) studies were used to determine drug-polymer interactions and drug entrapment. Entrapment efficiency, drug loading (DL), particle size, and zeta potential of nanoparticles were also studied. The $2^{3}$ factorial designs of experiments by Design-Expert ${ }^{\circledR} \mathrm{V} 9$ were used to optimize the particle size and entrapment efficiency of nanoparticles.

Results: The optimized batch had shown the entrapment efficiency of $98.10 \pm 0.24 \%$ and DL of $69.32 \pm 0.44 \%$ with particle size and zeta potential as $344 \pm 5 \mathrm{~nm}$ and $-42 \pm 11.40 \mathrm{mV}$, respectively. DSC, FTIR, and XRD studies confirmed the drug entrapment within nanoparticle matrix. SEM results showed spherical-shaped particles. Sustained release of drug from the nanoparticles was observed for 24 hrs period. Respirable fraction up to $52.37 \pm 0.7 \%$ demonstrates the formulation suitability for deep lung delivery. Lung inflammatory study showed a less inflammatory response.

Conclusion: Ionotropic gelation method can be used to prepare biocompatible particles with a high entrapment efficiency, DL, optimum particle size, and controlled release characteristics, which can serve as a convenient delivery system for D-CS and could be a potential alternative to the existing conventional therapy in MDR-TB.

Keywords: Nanoparticles, Alginate, Chitosan, Inhalation, Sustained release, Tuberculosis.

(C) 2016 The Authors. Published by Innovare Academic Sciences Pvt Ltd. This is an open access article under the CC BY license (http://creativecommons. org/licenses/by/4. 0/) DOI: http://dx.doi.org/10.22159/ajpcr.2016.v9s2.11814

\section{INTRODUCTION}

Mycobacterium tuberculosis (MTB) is second, next to HIV, in causing death from infectious diseases affecting one-third of the world population [1]. The current therapeutic management of MTB is inadequate due to non-compliance, lengthy course of treatment, and drug-related side effects, resulting in multi-drug resistant (MDR), extremely drug resistant [2], and even totally drug-resistant strains of TB, which are considerably harder to treat [3] and results in high dose requirements and subsequent intolerable toxicity. Nanoparticulate carrier systems may provide a possible solution to the problems associated with the treatment of $\mathrm{TB}$ with conventional drug delivery systems for antitubercular drugs (ATDs) [4]. Nanoparticles consisting of synthetic biodegradable polymers, natural biopolymers, lipids, and polysaccharides have been developed and tested over the past decades [5]. A wide range of compounds including aminoglycosides [6], antiviral drugs [7], b-lactam antibiotics [8], antifungals [9], and ATDs [10] have been successfully formulated into natural carrier systems. As far as ATDs are concerned, lipid-based formulations [11] as well as polymer-based formulations such as alginate (ALG) $[12,13]$ have been developed with proven therapeutic efficacy in experimental TB.

Properties of polymeric nanoparticles (PNPs) such as sustained release behavior can improve drug bioavailability and reduce dosing frequency and thereby help in improving patient compliance by resolving the problems of non-adherence to prescribed therapy which act as one of the major obstacles in the control of TB epidemics [4]. In addition, nanocarriers provide flexibility of drug administration via different routes such as oral, parenteral, nasal, and pulmonary [14]. A further advantage of this delivery system is its non-toxicity permitting the repeated administration of therapeutic agents. Chitosan-ALG (CHI-ALG) microspheres or beads have been widely studied for the entrapment of several drugs, proteins, cells, and oligonucleotides, with promising results [15-20].

Thus, in the present study, D-cycloserine (D-CS), a second-line agent for active pulmonary and extrapulmonary TB, is encapsulated into ALG-CHI nanoparticles by an ionotropic gelation method. Biodegradable PNs of D-CS were designed and optimized using $2^{3}$ factorial design to study the influence of formulation variables on particle size and entrapment efficiency of PNs. The process of formulation development involves a vital understanding of the effect of formulation factors, namely drug:polymer ratio on the properties of the formulation (response) such as particle size and entrapment efficiency. The development of D-CS-loaded PNs was optimized for their use in effective delivery of the drug. The aim of the present study is to prepare particles with favorable size, high entrapment efficiency, controlled release characteristics, in vivo tolerability, and aerodynamic characteristics, which can serve as a convenient delivery system for D-CS which has the potential for intermittent therapy of MDR-TB.

\section{MATERIALS AND METHODS}

Commercial grade D-CS was provided by Maneesh Pharmaceuticals, Mumbai, pharmaceutical grade of sodium ALG, manucol LKX was provided by Signet Chemicals, Mumbai, CHI, $85 \%$ deacylated was provided by theCentral Institute of Fisheries Technology (CIFT), Kochi, and calcium chloride was purchased from S. D. Fine Chemicals, Mumbai. Glacial acetic acid (A.R) was purchased from Merck, India. Distilled and Millipore water and all the excipients used were of analytical grade. 
Preparation of D-CS-loaded nanoparticles

D-CS-loaded ALG-CHI nanoparticles were prepared in a modified twostep procedure based on the ionotropic pre-gelation of polyanion with calcium chloride followed by polycationic cross-linking [21] (Fig. 1) A volume of $1 \mathrm{ml}$ of $18 \mathrm{mM}$ calcium chloride solution was dropped under ultrasonication at $100 \%$ amplitude for 2 minutes into a beaker containing $20 \mathrm{ml}$ of $0.063 \% \mathrm{w} / \mathrm{w}$ ALG solution to provide an ALG pre-gel. D-CS was mixed with the ALG solution before adding calcium chloride. A volume of $4 \mathrm{ml}$ of $0.05 \% \mathrm{CHI}$ solution was added dropwise into the pre-gel for 2 minutes under ultrasonication at $100 \%$ amplitude. The $\mathrm{pH}$ of ALG and CHI solutions was initially set to 5.0 and 5.5, respectively. A colloidal dispersion was obtained upon polycationic $\mathrm{CHI}$ addition, visible as the Tyndall effect. Nanoparticles were stirred for 30 minutes to improve curing, and drug-loaded nanoparticles were recovered by centrifugation at 20,000 rpm for 45 minutes and washed thrice with Millipore water. The final products were re-suspended and lyophilized (Labocon model LFD-BT-103) using 5\% w/v D-(+)-trehalose dihydrate as a cryoprotectant and then stored for future use.

\section{Optimization by factorial design method}

Design-Expert ${ }^{\circledR}$ Software Version 9.0.4.1 Stat-Ease Inc., Minneapolis, USA, was used for the generation and evaluation of the statistical experimental design [22-24]. The $2^{3}$ factorial design with three center points was used for the optimization of formulations of D-CS-loaded ALG-CHI nanoparticles. The independent variables such as the $\mathrm{CHI}$ concentration, sodium ALG concentration, and drug concentration were studied at two levels, low level (-1) and high level (+1), as shown in Table 1. The percentage entrapment efficiency and particle size (nm) were the dependent variables used in the design as shown in Table 2. The factorial design batches and responses are shown in Table 3.

\section{Evaluation of nanoparticles}

\section{Entrapment efficiency}

This parameter was calculated to know the amount of drug entrapped in the nanoparticles. Free unentrapped drug was separated from D-CS-loaded ALG-CHI nanoparticles by centrifugation at 20,000 rpm for 30 minutes. The supernatant was analyzed by ultraviolet (UV) spectrophotometer at $226 \mathrm{~nm}$ after required dilution. The entrapment efficiency was calculated by the indirect method as follows:

Entrapment efficiency $(\%)=($ Total drug-free drug $) /($ total drug $) \times 100$

\section{Drug loading (DL) efficiency}

The known amount of nanoparticles were taken, and the phosphate buffer saline (PBS) pH 7.4 was added into it for the lysis of the nanoparticles. The amount of drug in the supernatant was determined, and the total encapsulated drug in the nanoparticles was calculated. The DL was calculated as follows:

DL efficiency $(\%)=($ Total drug encapsulated into nanoparticles $) /($ total mass of nanoparticles $) \times 100$.

\section{Particle size and zeta potential analysis}

Particle size and zeta potential measurement was carried out using Nanosight NS500 computerized with nanoparticle tracking analysis (NTA), namely NTA 2.3 equipped with automated sample introduction, robotic motorized stage attached with red laser, and mounted on a CCD camera [25-27].

\section{Particle morphological study}

Shape and surface morphology of PNPs were done using scanning electron microscopy (SEM) [22,24]. For SEM analysis, the D-CS-loaded ALG-CHI nanoparticles were mounted directly on the Quanta 200 ESEM (FEI, USA) SEM aluminum stub, using double-sided sticking tape and scanned in a low vacuum chamber with a focused electron beam at $20 \mathrm{kV}$ and magnification of $12,000 \mathrm{X}$. Secondary electrons, emitted from the samples, were detected as the image formed.

\section{Differential scanning calorimetry (DSC)}

The DSC analysis was carried out using the Mettler DSC 60 computerized with Mettler Toledo Star software system with an intra-cooler (DSC Mettler STAR SW 9.20, Switzerland). Indium standard is used to

Table 1: Factors selected for optimization

\begin{tabular}{lllllll}
\hline Factor & Name & Units & Type & Low actual & High actual & Low-coded \\
\hline A & CHI & mg & Numeric & -1.00 & 1.00 & -1.00 \\
B & Sodium ALG & mg & Numeric & -1.00 & 1.00 & -1.00 \\
C & Drug & mg & Numeric & -1.00 & 1.00 & -1.00 \\
\hline
\end{tabular}

ALG: Alginate, CHI: Chitosan

Table 2: Response selected for optimization

\begin{tabular}{llllllll}
\hline Response & Name & Units & Obs & Analysis & Min & Max & Mean \\
\hline Y1 & Particle size & $\mathrm{nm}$ & 11 & Factorial & 176.000 & 441.000 & 324.636 \\
Y2 & Entrapment efficiency & $\%$ & 11 & Factorial & 97.580 & 99.210 & 98.263 \\
\hline
\end{tabular}

Table 3: Design matrix and responses

\begin{tabular}{|c|c|c|c|c|c|}
\hline Formulation code & $\begin{array}{l}\text { Factor A CHI } \\
(\mathrm{mg})\end{array}$ & $\begin{array}{l}\text { Factor B sodium ALG } \\
(\mathrm{mg})\end{array}$ & $\begin{array}{l}\text { Factor C drug } \\
\text { (mg) }\end{array}$ & $\begin{array}{l}\text { Response } 1 \text { particle } \\
\text { size }(\mathrm{nm})\end{array}$ & $\begin{array}{l}\text { Response } 2 \text { entrapment } \\
\text { efficiency }(\%)\end{array}$ \\
\hline F1 & 1.00 & -1.00 & -1.00 & $263 \pm 4$ & $98.07 \pm 0.53$ \\
\hline $\mathrm{F} 2$ & 1.00 & 1.00 & 1.00 & $344 \pm 5$ & $98.10 \pm 0.24$ \\
\hline F3 & -1.00 & -1.00 & 1.00 & $366 \pm 3$ & $97.69 \pm 0.47$ \\
\hline $\mathrm{F} 4$ & -1.00 & 1.00 & -1.00 & $441 \pm 2$ & $97.93 \pm 0.24$ \\
\hline F5 & 1.00 & -1.00 & 1.00 & $176 \pm 4$ & $98.02 \pm 0.54$ \\
\hline F6 & 0.00 & 0.00 & 0.00 & $377 \pm 6$ & $99.16 \pm 049$ \\
\hline F8 & 0.00 & 0.00 & 0.00 & $383 \pm 5$ & $99.21 \pm 0.54$ \\
\hline F9 & -1.00 & 1.00 & 1.00 & $246 \pm 4$ & $97.58 \pm 0.67$ \\
\hline F10 & -1.00 & -1.00 & -1.00 & $217 \pm 3$ & $97.96 \pm 0.81$ \\
\hline F11 & 0.00 & 0.00 & 0.00 & $372 \pm 2$ & $99.13 \pm 0.72$ \\
\hline
\end{tabular}

Low level (-1), medium level (0) and high level (+1): CHI (mg) $1(-1), 2(0)$ and $3(+1)$, sodium ALG (mg) $8(-1), 12(0)$ and $16(+1)$, drug (mg) $40(-1)$, $80(0)$ and 120 (+1). ALG: Alginate, CHI: Chitosan 
calibrate the instrument prior to use. Samples were accurately weighed and placed in aluminum sample pans. Samples are heated at a constant rate of $10^{\circ} \mathrm{C} /$ minute to get a thermogram. A dry purge of nitrogen gas $(20 \mathrm{ml} /$ minute $)$ was used for all runs. Samples were heated from $30^{\circ} \mathrm{C}$ to $350^{\circ} \mathrm{C}$. An empty aluminum pan was used as a reference. Scans were obtained from the samples. The DSC graphs are observed for the melting point and peak maxima [28].

\section{Fourier-transformed infrared (FTIR) spectroscopy}

The study was carried out to find the drug and excipient interactions and also to determine the entrapment of drug into the nanoparticles. FTIR spectrophotometer (Shimadzu, Japan) along with IR solution software (Version 1.4) was used to record FTIR spectra using $\mathrm{KBr}$ disc method. FT-IR transmission spectra were recorded by scanning $\mathrm{KBr}$ disc at $4 \mathrm{~mm} / \mathrm{s}$ at a resolution of $2 \mathrm{~cm}$ over a wavenumber range of $400-4000 / \mathrm{cm}$. The samples were observed for their characteristic peaks in the wavenumber region $[28,29]$.

\section{Powder X-ray diffraction (XRD) study}

XRD studies provide information about the crystallinity of the sample, which is reflected by a characteristic fingerprint region in the diffraction pattern. Studies were carried out using Philips Expertpro MPD diffractometer (PAN Analytical Inc., Germany), with a resolution of $0.001 \mathrm{~A}^{\circ}$. The samples stick over the glass slide by applying vacuum grease. A $\sim 0.5 \mathrm{~mm}$ layer is made by sprinkling $10 \mathrm{mg}$ of a sample over the slide. A copper target tube was used to radiate the sample which was exposed to all lines $(\lambda-1.54056)$. Scanning angles ranged from $5^{\circ}$ to $40^{\circ}$ of $2 \theta$. The current used was $30 \mathrm{~mA}$ and voltage was $40 \mathrm{kV}$.

\section{In vitro release study}

The in vitro release study was done to ensure the release of drug from nanoparticulate polymeric matrix [30]. The study was carried out by dissolving $5 \mathrm{mg}$ of freeze-dried D-CS-loaded ALG-CHI nanoparticles in $3 \mathrm{ml}$ PBS pH 7.4 which was added into dialysis membrane bag (MW cutoff 12,000, Himedia, India), clipped, and immersed into a beaker containing $300 \mathrm{ml}$ PBS pH 7.4. The temperature was maintained at $37^{\circ} \mathrm{C} \pm 0.1^{\circ} \mathrm{C}$ for the entire system, and continuous magnetic stirring at a rotation speed of $100 \mathrm{rpm}$ is maintained. At selected time intervals, $1 \mathrm{ml}$ of the release medium was removed from the system and replaced with $1 \mathrm{ml}$ buffer solution for maintaining sink condition. The amount of released drug in the medium was quantified by UV spectrophotometer (Jasco, Japan) at $226 \mathrm{~nm}$, and the percentage release of the drug at appropriate time intervals is calculated. The test was done in triplicate.

\section{Particle aerodynamic study}

Twin stage impinger is used to study the aerodynamic characteristics of aerosol generated from nanoparticulate suspension [24]. The suspension was made by dissolving $5 \mathrm{mg}$ of freeze-dried D-CSloaded ALG-CHI nanoparticles in $2 \mathrm{ml}$ of $0.9 \%$ sodium chloride. The redispersed nanoparticles are subjected to the holding chamber of compressed air nebulizer (Omron) which was connected to the mouth of the TSI through an adapter. The other end of the TSI was connected to a vacuum pump through a rotameter in-between, which was used to maintain a flow rate of $60 \mathrm{~L} /$ minute. A cutoff aerodynamic diameter of $6.4 \mu \mathrm{m}$ is maintained with this flow rate. $1 \mathrm{~N} \mathrm{HCl}$ of about $7 \mathrm{~mL}$ and $30 \mathrm{~mL}$ was filled in the upper stage (Stage 1) and lower stage (Stage 2) of the impinger, respectively. The nebulization continues till the chamber dryness and cessation of the aerosol generation. The stages of TSI are washed with $1 \mathrm{~N} \mathrm{HCl}$, and the final volume of Stage 1 and Stage 2 was made to $22 \mathrm{ml}$ and $45 \mathrm{ml}$, respectively. Drug deposition in different stages of TSI was determined by sampling at each stage and quantification through UV-visible spectroscopy (Jasco, Japan). The studies are carried out in triplicate. The aerodynamic characteristics of the nanoparticulate suspension were evaluated based on the parameters such as nebulization efficiency [31] and respirable fraction (RF) [32]. Percent fine particle fraction was calculated as the ratio of fine particle dose (FPD), i.e., drug deposited in Stage 2 to recovered dose (RD), i.e., drug deposited in Stage 1 and Stage 2, expressed as a percentage.

\section{In vivo lung inflammatory response studies}

This study was done to determine the inflammatory response of the D-CS-loaded ALG-CHI nanoparticles in Wistar rats. The study protocol was approved by the Institutional Animal Ethics Committee and the Committee for the Purpose of Control and Supervision of Experiments on Animals (CPCSEA), Regd. No. 25/1999/CPCSEA.

\section{Biochemical study}

The supernatant of the BALF was used to determine lactate dehydrogenase (LDH) release and total protein (TP) as markers for cell damage. Cell damage suggests inflammatory response and thereby biosafety of these nanoparticles can be determined by their measurement [33]. Thus, 12 Wistar rats were randomly divided into two groups with six animals in each group and they are treated in the following way: Group I: $0.9 \%$ sodium chloride was nebulized. Group II was nebulized with D-CS-loaded ALG-CHI nanoparticles. In vivo lung inflammatory response of D-CS-loaded ALG-CHI nanoparticles will be studied from nebulized formulations. For in vivo studies, a group of animals will be nebulized using the fabricated nose-only inhalation chamber, the drug formulations containing D-CS-loaded ALG-CHI nanoparticles suspended in $2 \mathrm{~mL}$ of $0.9 \%$ sodium chloride via a compressor nebulizer (Omron) with an exposure time of 10 minute/animal. The suspension will be placed inside the nebulizer's medicine cup and dosed in the inhalation chamber. After $4 \mathrm{hrs}$, all the animals are sacrificed and the lungs are taken for biochemical study and histopathological study, respectively. Bronchoalveolar lavage fluid was collected, and the estimation of inflammatory factors was performed using the standard procedures given in LDH kit (Coral Clinical Systems, India) and TP kit (Span Diagnostic Ltd, India).

\section{Lung tissue histopathology}

An ultrastructural change in the histology of lung tissues upon exposure to the D-CS-loaded ALG-CHI nanoparticles was carried out by preliminary histopathological evaluation. Lung tissues are stored in $10 \%(\mathrm{v} / \mathrm{v})$ neutral-buffered formalin for $24 \mathrm{hrs}$ at $4^{\circ} \mathrm{C}$. The lung tissues were embedded in paraffin for fixing. Control animals' lung section was prepared by the same procedure, but without application of the formulation. Sections of $4 \mu \mathrm{m}$ thickness were cut and stained with hematoxylin and eosin. These samples were then observed under a light microscope and compared with the control sample which was treated with $0.9 \%$ sodium chloride for any histological changes in tissue [33].

\section{Stability studies}

The stability study of lyophilized D-CS-loaded ALG-CHI nanoparticles was carried out according to the ICH guideline Q1A (R2) [34]. Long-term stability studies were carried out at refrigerated condition $\left(5^{\circ} \mathrm{C} \pm 3^{\circ} \mathrm{C}\right)$, at room temperature $\left(25^{\circ} \mathrm{C} \pm 2^{\circ} \mathrm{C} / 65 \pm 5 \% \mathrm{RH}\right)$ and at accelerated condition $\left(40^{\circ} \pm 2^{\circ} \mathrm{C} / 75 \pm 5 \% \mathrm{RH}\right)$ for 6 months. After each time interval, samples (1 mg) were withdrawn, rehydrated, and the particle size and percentage of D-CS remained in the nanoparticles were determined to assess the storage stability.

\section{RESULTS AND DISCUSSION}

\section{Optimization by factorial design method}

The $2^{3}$ factorial design with three center points was used for the optimization of formulations of D-CS-loaded ALG-CHI nanoparticles. The independent variables such as the CHI concentration, sodium ALG concentration, and drug concentration were studied at two levels which have significantly influenced the particle size and drug entrapment efficiency which is very much evident from the results as shown in Table 3 that represent the various combinations of independent variables with its resultant effect on the dependent variable.

By applying ANOVA at 0.05 levels, the effects of the independent variables $(A, B$, and $C)$ on the particle size $(\mathrm{nm})$ and entrapment efficiency (\%) were evaluated, and the following models were obtained. 
Model analysis for particle size

As shown in Table 4, a significant model has been obtained with model F-value of 282.24 and this large value could occur with only $0.35 \%$ due to noise. Significant model terms are having values $<0.0500$ for " $p>$ F." In this case, the significant model terms are A, B, C, AB, AC, BC, and ABC. The non-significant model terms are having values $>0.1000$. A significant curvature in the design space was implied by "curvature F-value" of 377.64. The chance of occurring this large value of "Curvature F-value" due to noise is only $0.26 \%$.

The signal-to-noise ratio was measured by "Adeq Precision." A desirable ratio was $>4$. An adequate signal was indicated by a ratio of 53.194 . The design space can be navigated by this model.

The final equation was obtained for particle size in terms of:

Coded factors: Particle size $=+304.87-12.62 * \mathrm{~A}+49.38 * \mathrm{~B}-21.87 * \mathrm{C}+23$. $37^{*} A^{*} B-10.38^{*} A^{*} C-37.38^{*} B^{*} C+48.63^{*} A^{*} B^{*} C$.

Actual factors: Particle size = $+304.87500-12.62500^{*} \mathrm{C} \mathrm{H} \mathrm{I}+49.37500^{*} \mathrm{~s}$ o d i u m A L G - 21.8750 0* d r u g + 23.3750 $0^{*} \mathrm{CH} \mathrm{I}^{*} \mathrm{~s}$ o d i u m A L G $-10.37500 * \mathrm{C} \mathrm{H} \mathrm{I}$ * D r u g - 37.3750 0 *s o d i u m ALG*Drug+48.62500*CHI*sodium ALG*drug.

\section{Model analysis for drug entrapment efficiency}

As shown in Table 5, a significant model has been obtained with model F-value of 21.81 and this large value could occur with only $4.45 \%$ due to noise. Significant model terms are having values $<0.0500$ for " $\mathrm{p}>\mathrm{F}$." In this case, the significant model terms are A, C, and AC. The non-significant model terms are having values $>0.1000$. A significant curvature in the design space was implied by the "curvature F-value" of 2063.61. The chances of occurring this large value of "Curvature F-value" due to noise is only $0.05 \%$.

Table 4: Response 1-Particle size: ANOVA for selected factorial model and analysis of variance table

\begin{tabular}{llllll}
\hline Source & $\begin{array}{l}\text { Sum of } \\
\text { squares }\end{array}$ & df & $\begin{array}{l}\text { Mean } \\
\text { square }\end{array}$ & F value & $\begin{array}{l}\text { p value } \\
\text { P>F }\end{array}$ \\
\hline Model & 59928.87 & 7 & 8561.27 & 282.24 & $0.0035 \mathrm{~S}$ \\
A-CHI & 1275.12 & 1 & 1275.12 & 42.04 & 0.0230 \\
B-sodium ALG & 19503.13 & 1 & 19503.13 & 642.96 & 0.0016 \\
C-drug & 3828.12 & 1 & 3828.12 & 126.20 & 0.0078 \\
AB & 4371.12 & 1 & 4371.12 & 144.10 & 0.0069 \\
AC & 861.13 & 1 & 861.13 & 28.39 & 0.0335 \\
BC & 11175.13 & 1 & 11175.13 & 368.41 & 0.0027 \\
ABC & 18915.13 & 1 & 18915.13 & 623.58 & 0.0016 \\
Curvature & 11455.00 & 1 & 11455.00 & 377.64 & $0.0026 \mathrm{~S}$ \\
Pure error & 60.67 & 2 & 30.33 & & \\
Cor total & 71444.55 & 10 & & & \\
\hline
\end{tabular}

ALG: Alginate, CHI: Chitosan, S: Significant
The signal-to-noise ratio was measured by "Adeq Precision." A desirable ratio was $>4$. An adequate signal was indicated by a ratio of 43.403 . The design space can be navigated by this model.

The final equation was obtained for entrapment Efficiency in terms of:

Coded factors: Entrapment efficiency $=+97.92+0.13 * \mathrm{~A}-0.011 * \mathrm{~B}-0.076$ ${ }^{*} \mathrm{C}+0.024^{*} \mathrm{~A}^{*} \mathrm{~B}+0.079 * \mathrm{~A}^{*} \mathrm{C} 3.750 \mathrm{E}-003^{*} \mathrm{~B}{ }^{*} \mathrm{C}+0.024^{*} \mathrm{~A}^{*} \mathrm{~B}{ }^{*} \mathrm{C}$.

Actual factors: Entrapment efficiency = $+97.92375+0.13375^{*} \mathrm{C} \mathrm{H} \mathrm{I}-0.011250^{*}$ s o d i u m A L G - 0.076250*C + 0.023750* C H I*s o d i u m $\mathrm{ALG}+0.078750^{*} \mathrm{CH} \mathrm{I}^{*} \mathrm{drug}+3.75000 \mathrm{E}-003^{*} \mathrm{~s} \mathrm{od} \mathrm{iu} \mathrm{m}$ $\mathrm{ALG}^{*}$ drug+0.023750*CHI*sodium ALG*drug.

\section{Response surface analysis}

The Design-Expert ${ }^{\circledR}$ 9.0.4.1 software was used to generate the threedimensional (3D) response surface graphs and related two-dimensional (2D) contour plots as shown in Figs. 2 and 3 [22,23,30]. The effects of the independent variables (factors) and their interactions are learned by $3 \mathrm{D}$ response surface graph while $2 \mathrm{D}$ contour plot provides a visual representation of response values. The particle size 3D response surface graphs are shown in Fig. 2a which depicted the increase in particle size $(\mathrm{nm})$ with the increasing polymer concentration while Fig. 3a depicting percentage entrapment efficiency was increasing with the decreasing polymer concentration. Nonlinear relationships are shown by the $2 \mathrm{D}$ contour plots (Figs. $2 \mathrm{~d}$ and $3 \mathrm{~d}$ ) relating to particle size $(\mathrm{nm})$ and entrapment efficiency (\%).

\section{Entrapment efficiency}

The effect of formulation variables such as concentrations of $\mathrm{CHI}$, sodium ALG, and drug on entrapment efficiency of D-CS-loaded ALG-CHI nanoparticles can be seen in 3D response surface graphs and 2D contour plot. As shown in Table 3, entrapment efficiency of these nanoparticles was found to vary between 97.69 and $99.21 \%$ (Fig. 2e and f). From response surface graphs, as shown in Fig. 2a, it can be seen that when the drug concentration remains same, the increase in the sodium ALG concentration from $0.06 \%$ led to the increase in entrapment efficiency, while concentration $<0.06 \%$ led to a decrease in entrapment efficiency. The highest entrapment efficiency of $99.21 \%$ was observed when the CHI, ALG, and the drug were used at intermediate concentrations. Varying CHI concentrations have shown no effect on entrapment efficiency as long as the sodium ALG concentration is lower than $0.06 \%$. The decrease in entrapment efficiency was seen when the higher concentration of drug was used due to the presence of the unentrapped drug in the formulation as shown in Fig. 2b. However, the concentration of sodium ALG above $0.06 \%$ led to the increase in entrapment efficiency whereas the concentration of sodium ALG remains constant. It has been observed from Fig. $2 \mathrm{c}$ that the lower concentration below the intermediate value of drug and CHI has shown an increase in entrapment efficiency while the higher concentration of both decreases the entrapment efficiency.

Table 5: Response 2-entrapment efficiency: ANOVA for selected factorial model and analysis of variance table

\begin{tabular}{lllll}
\hline Source & Sum of squares & df & Mean square & F value \\
\hline Model & 0.25 & 7 & 0.036 & 21.81 \\
A-sodium ALG & 0.14 & 1 & 0.14 & 87.62 \\
B-CHI & $1.013 \mathrm{E}-003$ & 1 & $1.013 \mathrm{E}-003$ & $0.0445 \mathrm{~S}$ \\
C-Drug & 0.047 & 1 & 0.047 & 0.0112 \\
AB & $4.513 \mathrm{E}-003$ & 1 & $4.513 \mathrm{E}-003$ & 0.5136 \\
AC & 0.050 & 1 & 0.050 & 2.76 \\
BC & $1.125 \mathrm{E}-004$ & 1 & $1.125 \mathrm{E}-004$ & 30.38 \\
ABC & $4.512 \mathrm{E}-003$ & 1 & $4.512 \mathrm{E}-003$ & 0.069 \\
Curvature & 3.37 & 1 & 3.37 & 0.2384 \\
Pure error & $3.267 \mathrm{E}-003$ & 2 & $1.633 \mathrm{E}-003$ & 0.0314 \\
Cor total & 3.62 & 10 & & 0.2384 \\
\hline
\end{tabular}

ALG: Alginate, CHI: Chitosan, S: Significant 


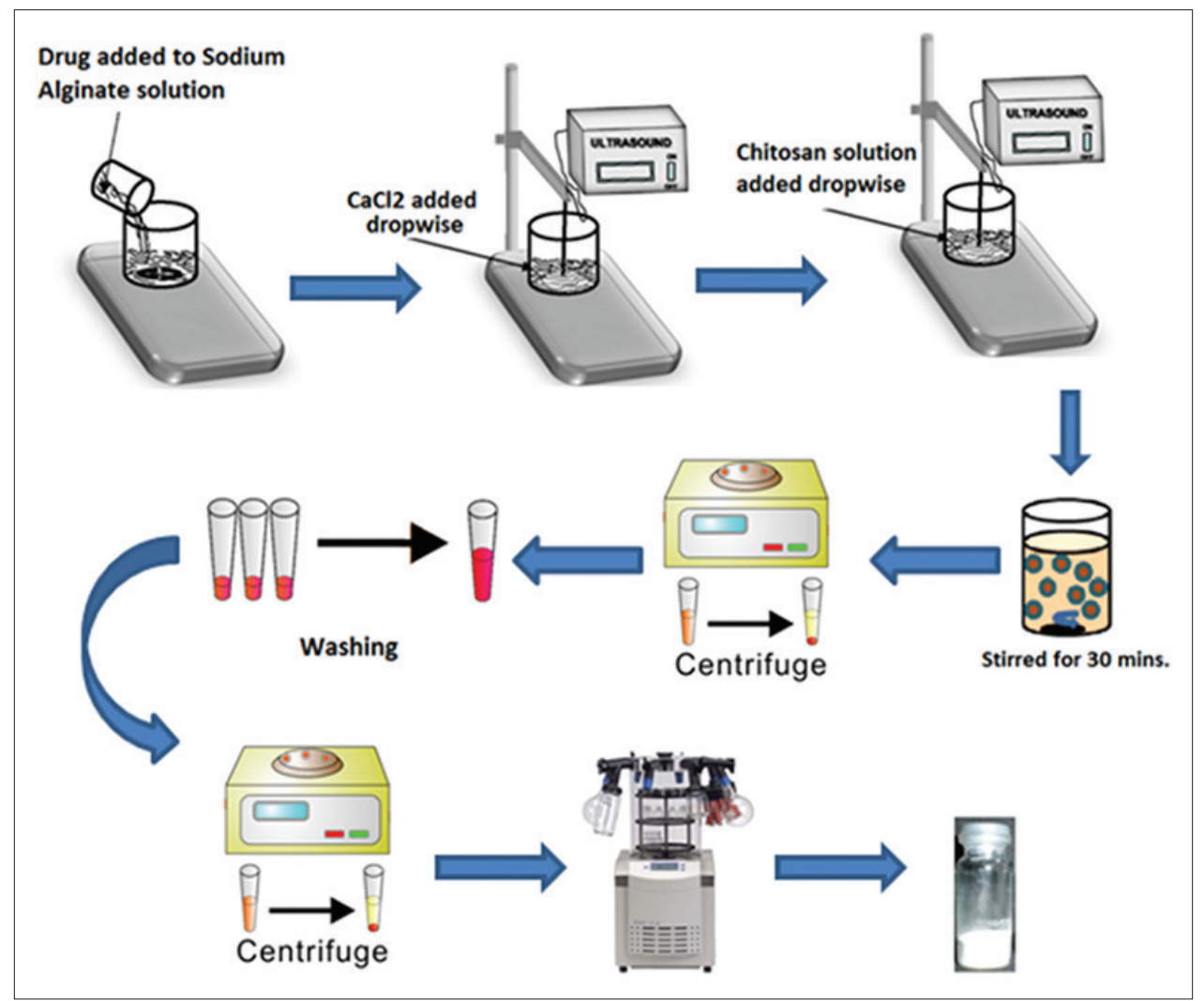

Fig. 1: Preparation method of D-cycloserine-loaded alginate-chitosan nanoparticles

\section{Particle size}

The average particle size of all nanoparticulate formulations was in the range of $176 \pm 4 \mathrm{~nm}$ to $441 \pm 2 \mathrm{~nm}$ as shown in Table 6 indicating that all the formulations exist in the nanometer range. NanoSight NS500 was the particle size analyzer used to measure the particle size of nanoparticles. This particle size analyzer works on the principle of NTA technology which is different from the dynamic laser scattering technology of particle size measurement. The particle size distribution obtained by the NTA is number distribution whereas dynamic light scattering (DLS) gives intensity distributions. The polydispersity of nanoparticles is measured by particle size's standard deviation (SD) in NTA technology such as polydispersity index (PDI) in DLS technology. Particle size's SD was in the range of $81 \pm 5-176 \pm 4 \mathrm{~nm}$ which determined the PDI ensuring some formulations with a homogeneous population of the nanoparticles (Fig. 3b). A relationship can be seen between the concentration of $\mathrm{CHI}$ and particle size in 3D response surface graphs and $2 \mathrm{D}$ contour plots. The increase in particle size was observed with the increase in CHI concentration, as seen in Fig. 3a and c. However, a flakes formation takes place with an increased amount of $\mathrm{CHI}$ in formulation [35]. Various researchers [36] have studied the effects of CHI and ALG mass ratio upon the characteristics of nanoparticles. The concentration of $\mathrm{CHI}$ and ALG affects the particle size of nanoparticles. It can be seen that when the $\mathrm{CHI}$ and ALG mass ratio was less, the highest particle size was observed while smaller particles were obtained at large $\mathrm{CHI}$ and ALG mass ratio as shown in Fig. 3a and c. These findings led to the assumption that when the functional groups of CHI and ALG are in stoichiometric proportion for interaction, a small size of nanoparticles is obtained.

\section{Optimization and model validation}

The selection of optimized formulation was done by numerical analysis of data from model generated by Design-Expert ${ }^{\circledR}$ 9.0.4.1 software. This software offers the possibility to simultaneously change each variable and gives possible optimum selections with their respective value of desirability. After studying the effect of independent variables on the
Table 6: Average particle size of the formulations of D-CS-loaded ALG-CHI nanoparticles with particle size's SD

\begin{tabular}{lll}
\hline Batch no. & Avg. particle size (nm) & Particle size's SD (nm) \\
\hline P1 & $263 \pm 4$ & $98 \pm 4$ \\
P2 & $344 \pm 5$ & $118 \pm 6$ \\
P3 & $366 \pm 3$ & $110 \pm 3$ \\
P4 & $441 \pm 2$ & $176 \pm 6$ \\
P5 & $176 \pm 4$ & $99 \pm 4$ \\
P6 & $377 \pm 6$ & $129 \pm 7$ \\
P7 & $386 \pm 3$ & $127 \pm 5$ \\
P8 & $383 \pm 5$ & $120 \pm 2$ \\
P9 & $246 \pm 4$ & $81 \pm 5$ \\
P10 & $217 \pm 3$ & $101 \pm 4$ \\
P11 & $372 \pm 2$ & $129 \pm 6$ \\
\hline
\end{tabular}

Mean \pm SD, n=3. D-CS: D-cycloserine, ALG: Alginate, CHI: Chitosan, SD: Standard deviation

responses, the levels of these variables that give the optimum response were determined. Hence, all the variables were decided in range, and the optimum formulation was the one that gives the optimum value of particle size along with a high amount of drug entrapment. By solving equations 1 and 2 simultaneously, analyzing response surface plots and constraints, the optimum levels for the concentration of drug, sodium ALG, and CHI were found to be $120 \mathrm{mg}, 16 \mathrm{mg}$, and $3 \mathrm{mg}$, respectively. Values of the variables for the optimized batch are shown in Table 7. The optimized batch had a desirability value of 0.720 which is as shown in Fig. 4. The optimization capability of model obtained from the data analysis of full $2^{3}$ factorial design was evaluated by preparing the D-CS-loaded optimized formulation according to the optimal process variable settings and evaluation of their particle size $(\mathrm{nm})$ and percentage entrapment efficiency. The optimized formulation was found to have the entrapment efficiency and particle size with values as $98.10 \pm 0.24 \%$ and $344 \pm 5 \mathrm{~nm}$, respectively. This reveals that mathematical models obtained from the full 23 factorial design were 


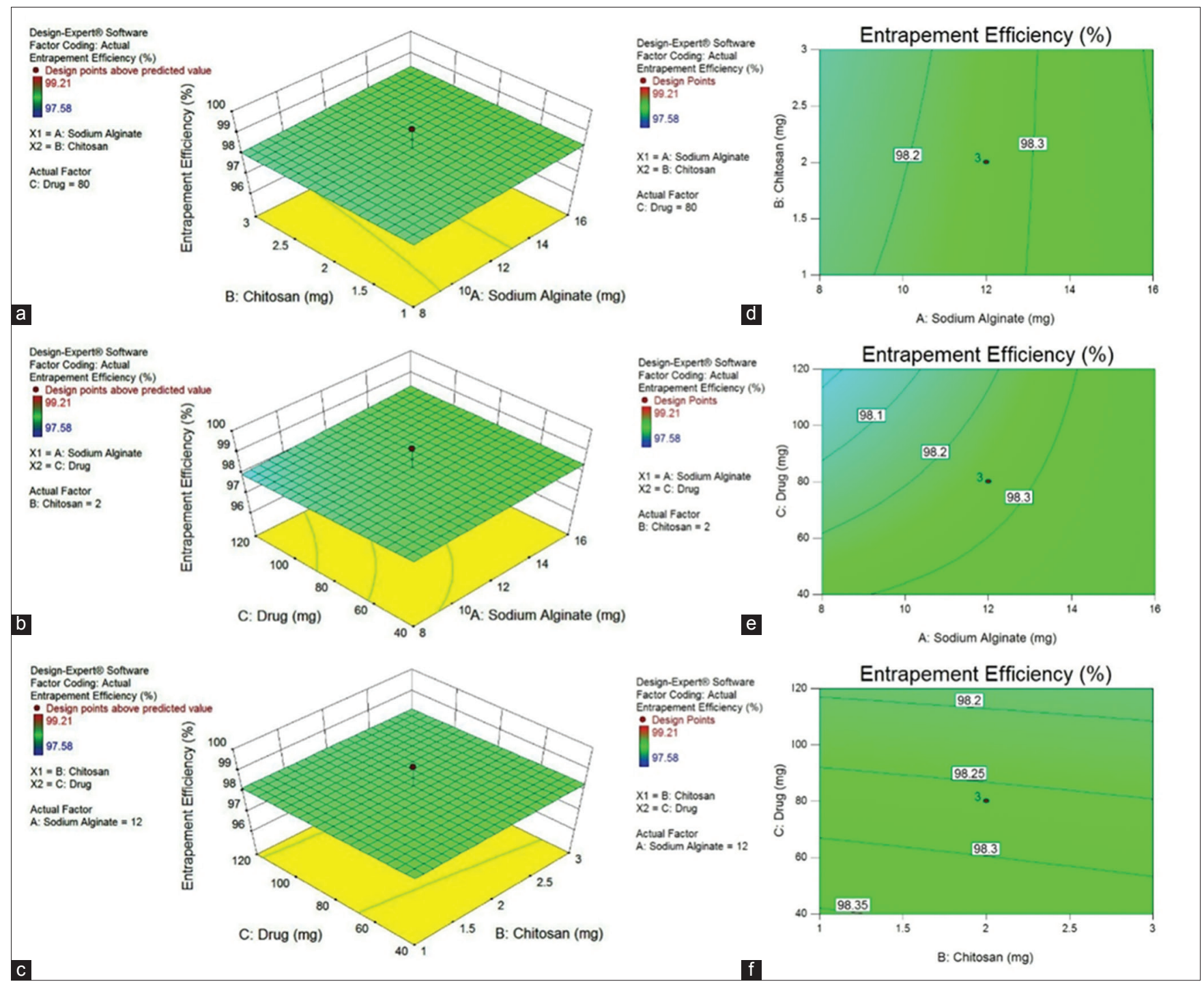

Fig. 2. (a, b, and c) three-dimensional response surface plot, (d, e, and f) two-dimensional contour plots showing the effect of different process parameters on the percentage entrapment efficiency

Table 7: Optimized formulation of ALG-CHI nanoparticles as per the Design-Expert ${ }^{\circledR}$ 9.0.4.1 software

\begin{tabular}{lllllll}
\hline Independent variables & Criteria & Value $\mathbf{( m g )}$ & Dependent variables & Criteria & Value & Desirability \\
\hline Drug & In range & 120 & Particle size & Maximum & $363.76 \mathrm{~nm}$ & 0.720 \\
Sodium ALG & In range & 16 & Entrapment efficiency & Maximum & $98.44 \%$ & \\
CHI & In range & 3 & & & & \\
\hline
\end{tabular}

ALG: Alginate, CHI: Chitosan

well fitted. The validity of $2^{3}$ factorial design for optimization of NPs, illustrated by the similarity of experimental values with predicted values, were within the percentage of predicted error zone and were in close agreement with the theoretical values as shown in Table 8. According to the desired criteria of optimum particle size and higher drug entrapment efficiency, the optimized batch was selected for further characterization.

\section{Evaluation of nanoparticles}

Entrapment efficiency

The entrapment efficiency of optimized batch of D-CS-loaded ALG-CHI nanoparticles was found to be $98.10 \pm 0.24 \%$. The ALG-CHI nanoparticles exhibited a very high entrapment efficiency due to the tightly packed structure of ALG-CHI nanoparticles.
Table 8: Comparison of predicted and experimental results for optimized ALG-CHI nanoparticles

\begin{tabular}{lll}
\hline Results & Particle size & Entrapment efficiency \\
\hline Predicted & $363.76 \mathrm{~nm}$ & $98.44 \%$ \\
Experimental & $344 \pm 5 \mathrm{~nm}$ & $98.10 \pm 0.24 \%$ \\
SD & 13.97242 & 0.24041 \\
\hline
\end{tabular}

ALG: Alginate, CHI: Chitosan, SD: Standard deviation

\section{DL efficiency}

DL of $69.32 \pm 0.44 \%$ was observed for the optimized batch of D-CSloaded ALG-CHI nanoparticles. The high DL helps in the deposition of high amount of drugs at the site of infection. 


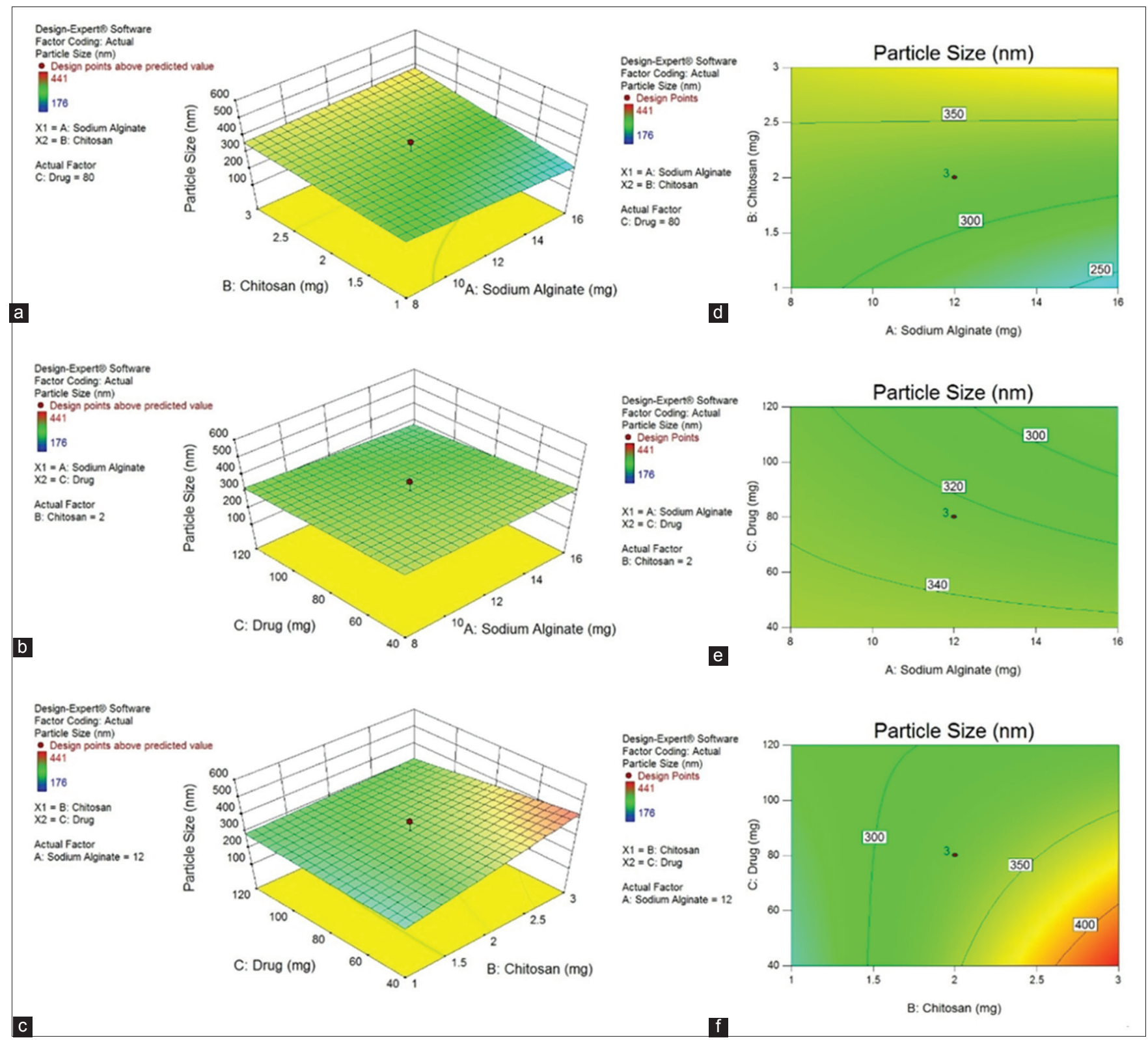

Fig. 3: (a, b and c) three-dimensional response surface plot, (d, e and f) two-dimensional contour plots showing the effect of different process parameters on the particle size

\section{Particle size and zeta potential analysis}

The optimized particle size of the formulation is $344 \pm 5 \mathrm{~nm}$ with the particle size's SD values of $118 \pm 6 \mathrm{~nm}$ as shown in Fig. 5. Particles per frame are shown in Fig. 6. The zeta potential of optimized batch of ALGCHI nanoparticles was found to be $-42 \pm 11.40 \mathrm{mV}$ as shown in Fig. 7 . The presence of ALG gives rise to such high negative value. The charge of the nanoparticles is an important parameter that can influence properties such as stability. The high negative zeta potential will prevent aggregation and increase the stability of nanoparticulate formulation.

\section{Particle morphological studies}

SEM imaging was used to confirm the formation of D-CS-loaded ALG-CHI nanoparticles and to visualize its size, shape, and surface morphology. SEM analysis confirmed that the particles formed using an ionotropic gelation were having desired particle size ranging $250-400 \mathrm{~nm}$ and also showed the spherical shape and smooth surface as shown in Fig. 8.

\section{DSC}

DSC is considered a tool to investigate the melting behavior of crystalline materials. DSC thermogram of D-CS showed the exothermic peak at $154.88^{\circ} \mathrm{C}$ which represents the melting point of the drug as shown in Fig. 9a. Sodium ALG showed an initial exothermic peak at $27.99^{\circ} \mathrm{C}$ and a higher endothermic peak at $241.91^{\circ} \mathrm{C}$ as shown in Fig. 9b. CHI revealed an endothermic peak at $62.81^{\circ} \mathrm{C}$ as shown in Fig. 9c. Endothermic peaks were correlated with the loss of water associated to hydrophilic groups of polymers while exothermic peaks arise due to dehydration and depolymerization reactions between the D-CS and the polymers in the solid state. Peaks of physical mixture appeared to be combinations of each material's peaks as shown in Fig. 9d. Thermogram of the ALG-CHI complexes was shifted from those of physical mixture. The characteristic exothermic peak for D-CS was found to be changed in intensity and shifted to $172.94^{\circ} \mathrm{C}$, probably because of interaction with polyelectrolyte complex and entrapment in CS-ALG nanoparticles. In addition, two characteristic exothermic peaks of ALG at $27.99^{\circ} \mathrm{C}$ and $241.91^{\circ} \mathrm{C}$ were found to disappear and could not be seen in D-CS-loaded ALG-CHI nanoparticles. Similarly, the exothermic peak of $\mathrm{CHI}$ was also found to be missing in the D-CS-loaded ALG-CHI nanoparticles thermogram. An exothermic peak at $282.29^{\circ} \mathrm{C}$ appeared in the nanoparticles thermogram as due to complexation of the polyelectrolytes resulted in a new chemical bond as shown in Fig. $9 \mathrm{f}$. 


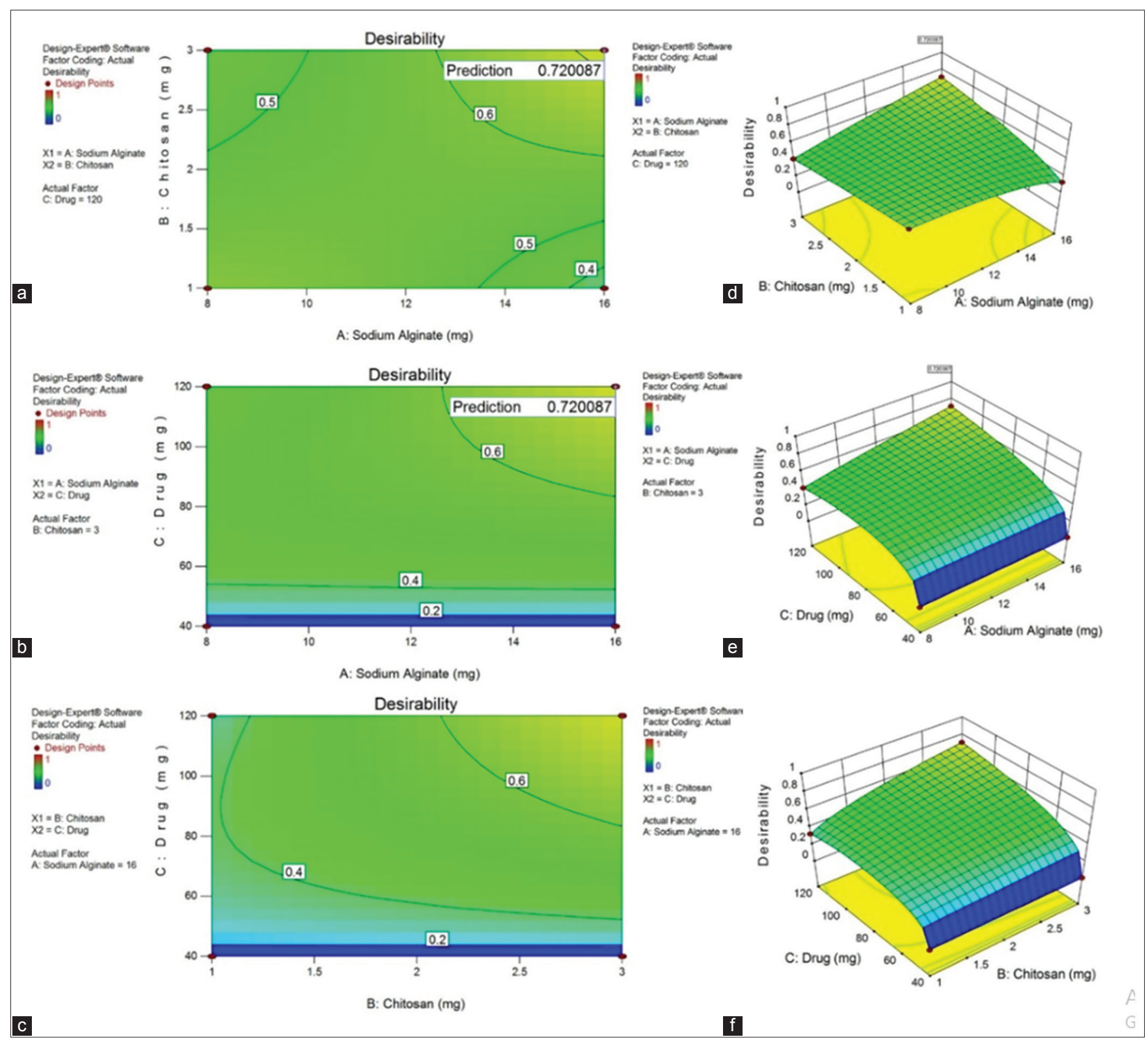

Fig. 4: (a, b, and c) two-dimensional contour plots, (d, e, and f) three-dimensional response surface plot showing the effect of different process parameters on the desirability

While blank nanoparticles lack the characteristic exothermic peak of the drug as desired as shown in Fig. 9e [28,30]. The peak type, peak temperatures, and enthalpy changes in the DSC thermograms of drug, polymers, and nanoparticles were shown in Table 9.

\section{FTIR spectroscopy}

FTIR was adopted to characterize the potential polyelectrolytes interactions and drug entrapment in the nanoparticulate systems. In addition, the potential intermolecular interactions between the polymers and drugs were analyzed by the FTIR spectra of ALG, CHI, D-CS, blank, and D-CS-loaded ALG-CHI nanoparticles as shown in Fig. 10. FTIR spectrum of ALG as shown in Fig. 10b had three characteristic peaks at 3376,3168 , and $2928 / \mathrm{cm}$, due to the hydroxyl group and $\mathrm{O}-\mathrm{H}$ stretching. The symmetric $\mathrm{COO}$ - and asymmetric COO- stretching vibration of carboxylate salt group shows peaks at 1612 and $1408 / \mathrm{cm}$, and peak at around $1036 / \mathrm{cm}$ shows C-O-C stretching due to ALG saccharide structure [37]. In the spectrum of $\mathrm{CHI}$, as shown in Fig. 10c, a broad peak at $3422 / \mathrm{cm}$ was observed due to the amine and hydroxyl groups while the $-\mathrm{OH}$ stretching represents a peak at $2848 / \mathrm{cm}$.
The sharp peak at 1642 corresponds to carbonyl $(\mathrm{C}=0)$ stretching of the $2^{\circ}$ amine and peak at $1555 / \mathrm{cm}$ represents a bending vibration of the $\mathrm{N}-\mathrm{H}$ group, $\mathrm{N}$-acetylated residues [38]. Peaks observed at 1436 and $1308 / \mathrm{cm}$ were due to the amide and ether bonds (N-H stretching) and amide III band ( $\mathrm{N}-\mathrm{H}$ stretching), respectively. The peaks shown at 1111 and $1022 / \mathrm{cm}$ are due to the secondary hydroxyl group and the primary hydroxyl group [39]. In the spectrum of blank ALG-CHI nanoparticles as shown in Fig. 10d, ALG peaks were slightly shifted to higher frequencies (1612 to $1668 / \mathrm{cm}$ and 1408 to $1440 / \mathrm{cm}$ ), and CHI amide peaks were converted into a singlet band at $1668 / \mathrm{cm}$ while the hydroxyl group peak, with the broad absorbance at $3436 / \mathrm{cm}$, can be seen in the formed nanoparticles. While in the spectrum of drug-loaded nanoparticles, we observed that the peaks at 1612 and $1408 / \mathrm{cm}$ are due to symmetric COO- and asymmetric COO- stretching vibration of carboxylate salt groups of ALG, which broaden and slightly shift from 1612 to 1668 and 1408 to 1416 after complexation with CHI. The characteristic amide peaks of $\mathrm{CHI}$ at 1642 changes into singlet band at 1668 whereas the amino peak at 1555 shifted to 1564 due to complexation of $\mathrm{CHI}$ with ALG. These changes in the peaks of the carboxyl groups, amino groups, 
Table 9: Peak temperatures and enthalpy changes in the DSC thermograms collected from drug, polymers, cross-linking agent, physical mixtures, drug-loaded nanoparticles, and blank nanoparticles

\begin{tabular}{|c|c|c|c|c|c|c|c|}
\hline \multirow[t]{2}{*}{ S. No } & \multirow[t]{2}{*}{ Ingredient } & \multirow[t]{2}{*}{ Peak type } & \multicolumn{3}{|c|}{ Temperature $\left({ }^{\circ} \mathrm{C}\right)$} & \multirow[t]{2}{*}{ Normalized $\Delta \mathbf{H}(\mathrm{J} / \mathrm{g})$} & \multirow[t]{2}{*}{ Integral mJ } \\
\hline & & & Onset & Peak & Endset & & \\
\hline 1 & D-CS & Exothermic & 150.03 & 154.88 & 157.55 & 1502.76 & 3907.17 \\
\hline \multirow[t]{2}{*}{2} & Sodium ALG & Exothermic & 25.80 & 27.99 & 29.48 & 162.14 & 393.99 \\
\hline & & Exothermic & 227.19 & 241.91 & 255.84 & 223.53 & 543.19 \\
\hline 3 & $\mathrm{CHI}$ & Endothermic & 26.75 & 62.81 & 105.23 & -428.09 & -1070.23 \\
\hline \multirow[t]{2}{*}{4} & Calcium chloride & Endothermic & 43.09 & 46.14 & 47.99 & -45.40 & -145.75 \\
\hline & & Endothermic & 134.80 & 167.79 & 173.22 & -604.50 & -1940.45 \\
\hline \multirow[t]{2}{*}{5} & Physical mixture & Endothermic & 114.71 & 122.09 & 132.38 & -53.10 & -178.41 \\
\hline & & Exothermic & 148.52 & 151.80 & 158.42 & 301.07 & 1011.58 \\
\hline \multirow[t]{2}{*}{6} & D-CS-loaded & Exothermic & 161.93 & 172.94 & 192.78 & 156.54 & 406.99 \\
\hline & nanoparticles & Endothermic & 248.61 & 282.29 & 297.27 & -152.20 & -395.72 \\
\hline \multirow[t]{2}{*}{7} & Blank nanoparticles & Endothermic & 70.10 & 108.93 & 124.06 & -819.93 & -2951.75 \\
\hline & & Exothermic & 247.98 & 266.03 & 276.95 & 54.44 & 195.99 \\
\hline
\end{tabular}

D-CS: D-cycloserine, DSC: Differential scanning calorimetry, ALG: Alginate, CHI: Chitosan

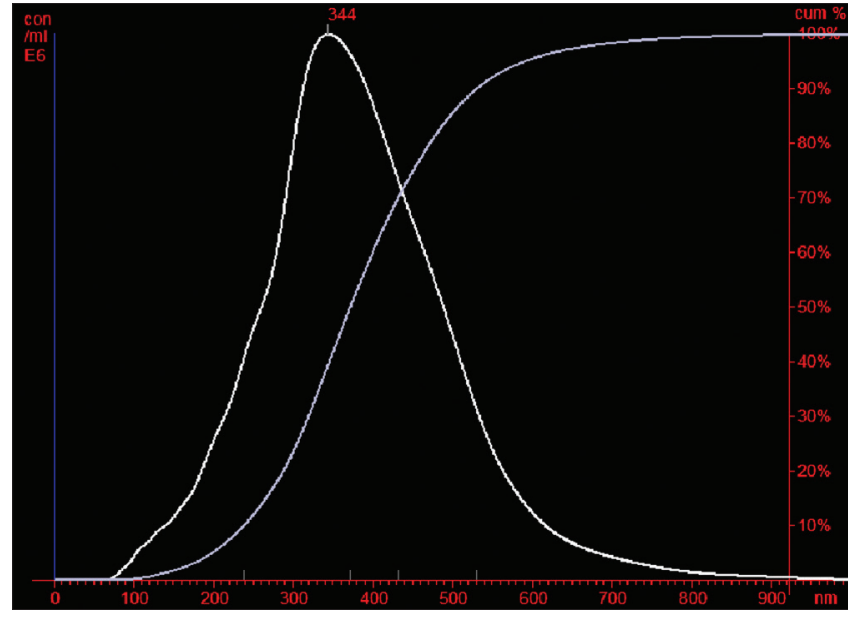

Fig. 5: The size distribution curve of D-cycloserine-loaded alginate-chitosan nanoparticles

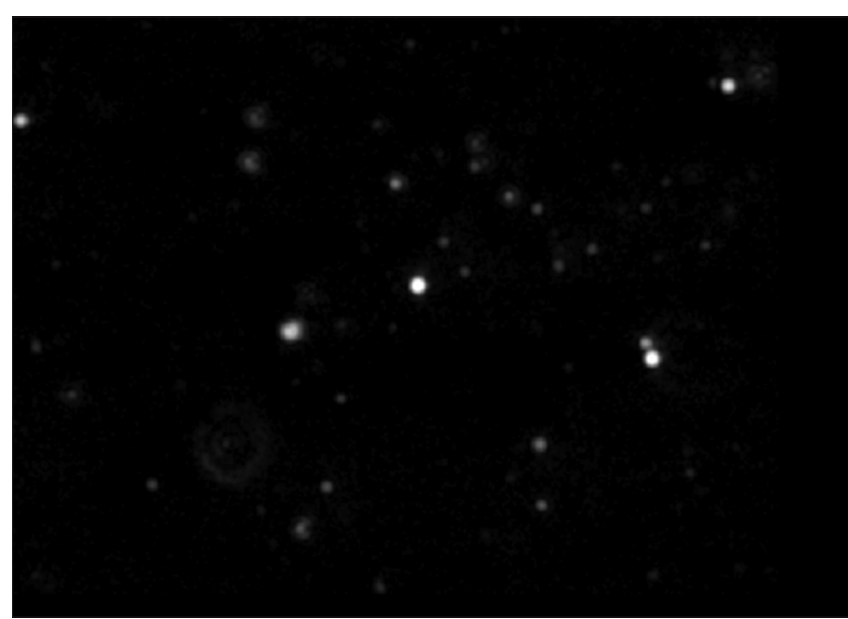

Fig. 6: Particle per video frame of D-cycloserine-loaded alginatechitosan nanoparticles

and amide bonds were due to ionic complexation between the $\mathrm{CHI}$ and ALG by interaction of the amino group and carboxyl group of the cationic and anionic polymers, respectively [36]. The broadband at $3422 / \mathrm{cm}$ corresponded to the amine and hydroxyl groups shift to 3448 . The unreacted amino (NH2-) groups of $\mathrm{CHI}$ shows a peak at $1642 / \mathrm{cm}$ in both the spectra of CHI and D-CS-loaded ALG-CHI nanoparticles.In

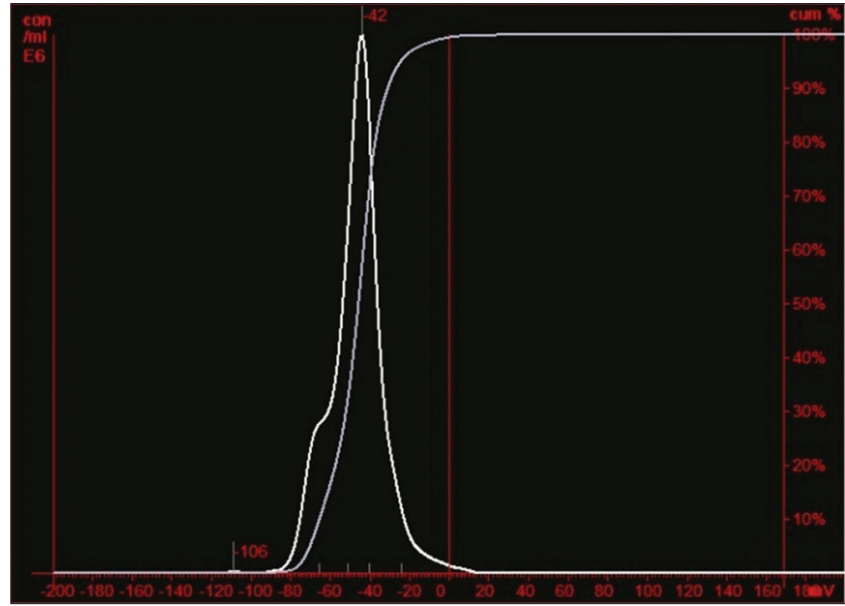

Fig. 7: Zeta potential of an optimized batch of alginate-chitosan nanoparticles

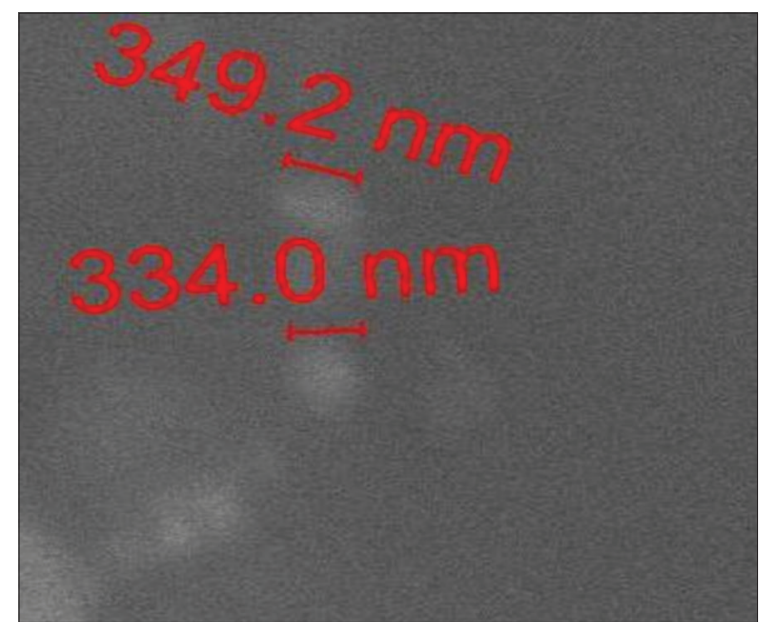

Fig. 8: Scanning electron micrographs image of D-cycloserineloaded polymeric nanoparticles

addition, the peak seen at $836 / \mathrm{cm}$ in ALG and D-CS-loaded ALG-CHI nanoparticles of FTIR spectra was due to unreacted $(-\mathrm{COOH})$ groups of ALG [22].

Pure D-CS displays peak characteristics as shown in Fig. 10a at 1523/cm corresponding to the absorption of resonance-stabilized hydroxamate 


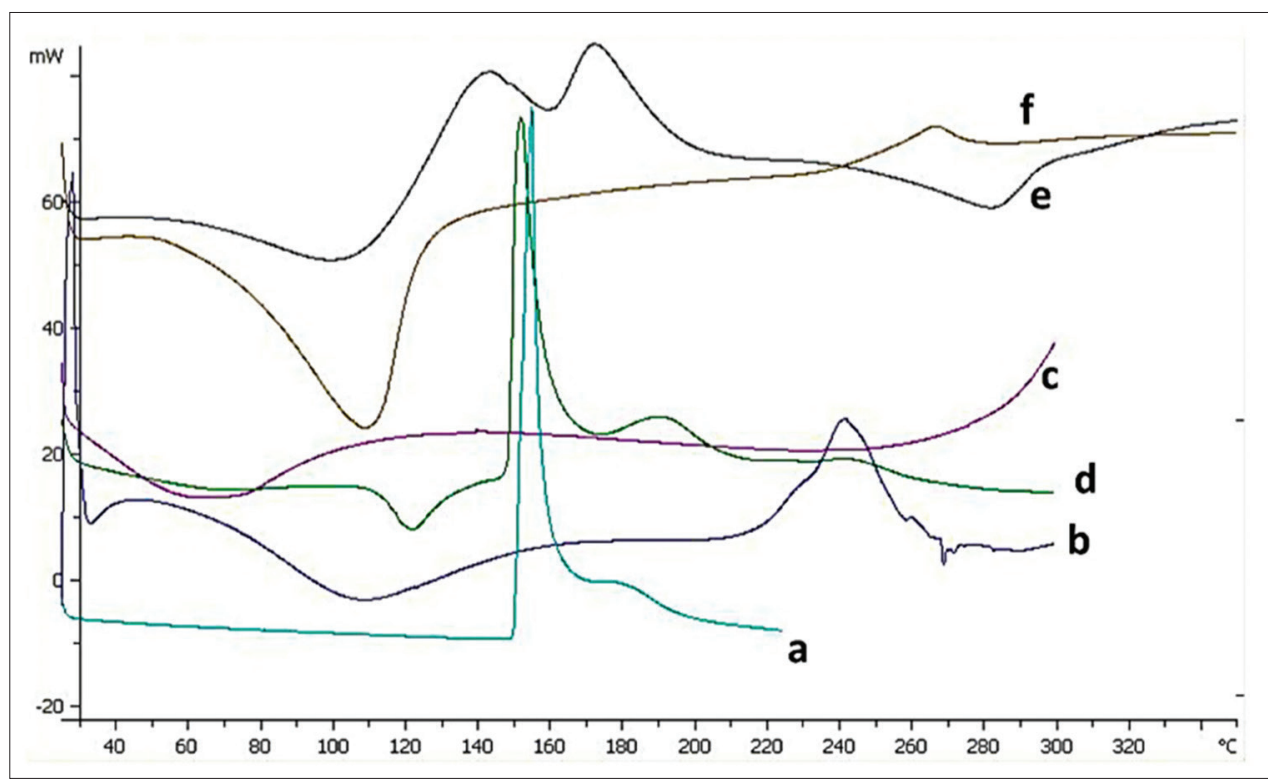

Fig. 9: Differential scanning calorimetry thermograms of (a) D-cycloserine (D-CS), (b) sodium alginate (ALG), (c) chitosan (CHI), (d) physical mixture, (e) blank ALG-CHI nanoparticles, and (f) D-CS-loaded ALG-CHI nanoparticles

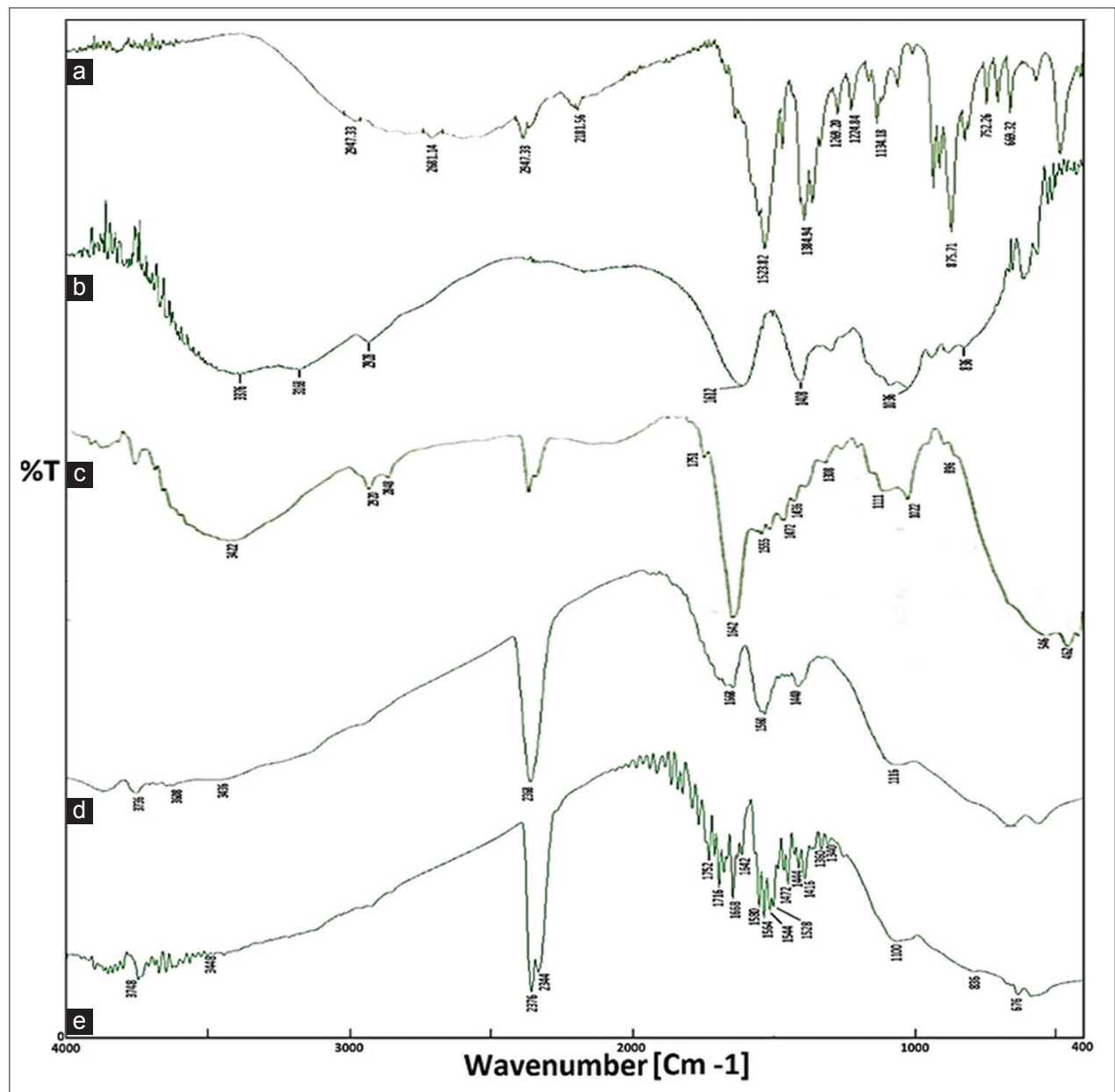

Fig. 10: Fourier-transformed infrared spectrum of (a) D-cycloserine (D-CS), (b) sodium alginate (ALG), (c) chitosan (CHI), (d) blank ALGCHI nanoparticles, and (e) D-CS-loaded ALG-CHI nanoparticles

anion. The asymmetric (-NH3) N-H band shows peak at $1630 / \mathrm{cm}$. The characteristic absorption bands between 3300-2100 are due to broad (-NH3) absorption = N-H stretch, extended by a combination of bands at $2181,2364,2681$, and 2947 , respectively. The peaks at 669,772 , and 800 correspond to $\mathrm{N}-\mathrm{H}$ out of the plane stretch. A characteristic peak at 500 was observed due to torsional (-NH3) $\mathrm{N}-\mathrm{H}$ oscillation. 
However, in the final spectrum of formulation (Fig. 10e), D-CS showed minor shifting of peaks to $1528 / \mathrm{cm}, 1642 / \mathrm{cm}, 2344 / \mathrm{cm}$, and $676 / \mathrm{cm}$ for absorption resonance stabilized hydroxamate anion, asymmetric (-NH3) N-H band, = N - H stretching, and $\mathrm{N}-\mathrm{H}$ out of plane stretching, respectively. D-CS appeared in the D-CS-loaded ALG-CHI nanoparticles, which probably indicate that the drug was entrapped in the polymeric matrix.

\section{Powder XRD study}

XRD patterns were determined for the drug, D-CS-loaded ALG-CHI nanoparticles, and physical mixture. XRD pattern for pure drug from $2 \theta$ showed distinctive sharp peaks approximately at $18.89^{\circ}, 19.59^{\circ}$, $20.99^{\circ}, 22.25^{\circ}, 23.64^{\circ}, 29.59^{\circ}, 31.78^{\circ}$, and $33.25^{\circ}$, indicating its crystalline nature. Physical mixture has found to have similar peaks of drug indicating no interaction with the polymers and crosslinkers. After ionic cross-linking with ALG, no peaks were found in the diffractogram of selected ALG-CHI nanoparticles, reflecting the destruction of the native $\mathrm{CHI}$ packing structure as shown in Fig. 11 [40]. D-CS-loaded ALG-CHI nanoparticles are composed of a network structure closely packed due to interpenetration and cross-linking of polymer chains to each other by ALG counter-ions. The XRD implicated greater disarray in chain alignment in the nanoparticles after cross-linking [41]. The characteristic peaks of the drug were found to be absent in the D-CSloaded ALG-CHI nanoparticles. None of the drug peaks could be seen by close examination of the diffractogram of representative nanoparticles. The absence of characteristic drug peak in the developed D-CS-loaded ALG-CHI nanoparticles indicates complete amorphization of the drug. This indicates that drug was molecularly dispersed into a PNP, and there could be less or no free drug in crystalline form on the surface of the nanoparticles. From this, it is evident that an XRD signal of encapsulated drug is very difficult to detect, which showed that the drug is dispersed at a molecular level in the polymeric matrix. The sharp peaks in the XRD patterns of D-CS-loaded ALG-CHI nanoparticles disappeared due to complexation reaction and less intensity peaks that appear due to the intermolecular interaction between drug and polymer matrix resulting in complex matrix [24]. Its sharp crystal peaks were overlapped with the noise of the polymers and disappeared, indicating that drug was completely and successfully encapsulated into the cores of ALG-CHI nanoparticles, confirming DSC and FTIR results. No other high-intensity peaks were seen in D-CS-loaded ALG-CHI nanoparticles, revealing the amorphous nature of the polymers and crosslinker.

\section{In vitro release study}

In vitro release properties of D-CS (Fig. 12) show the cumulative release curves of the drug from D-CS-loaded ALG-CHI nanoparticles at various $\mathrm{pH}$ at $37^{\circ} \mathrm{C} \pm 0.5^{\circ} \mathrm{C}$ as a function of time. In the in vitro release study of the optimized formulation in PBS, pH 7.4 (Fig. 12) showed a biphasic release pattern with initial burst release of about $34.49 \%$ of D-CS,

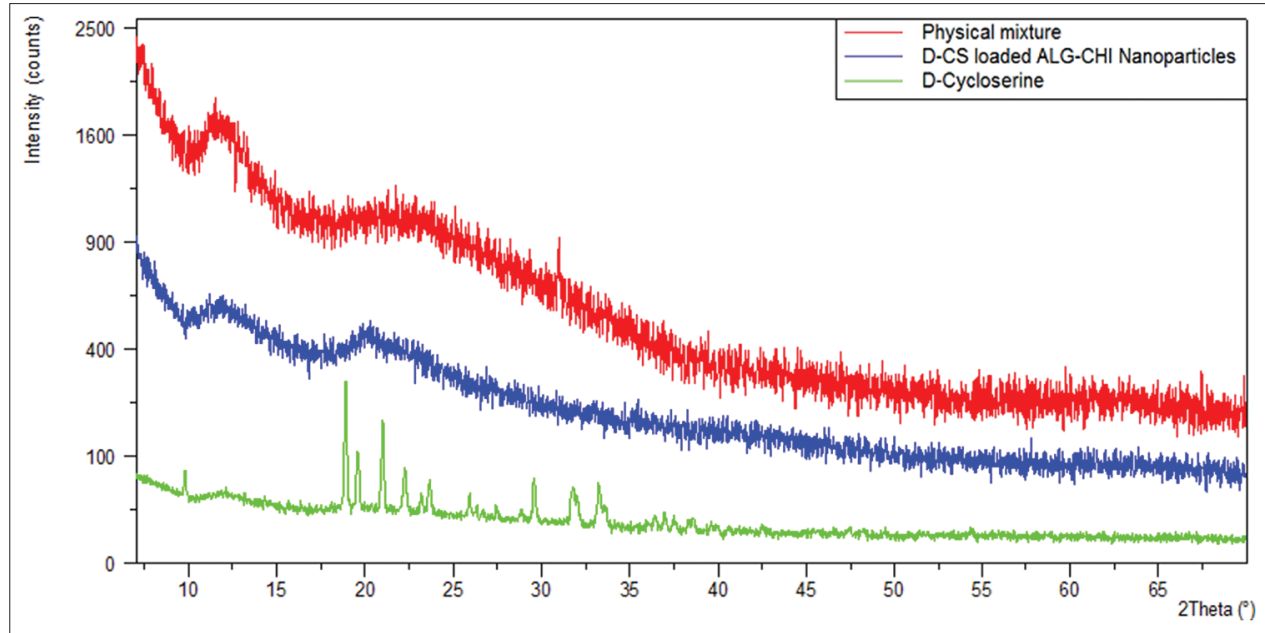

Fig. 11: X-ray diffractograms of D-CS (D-cycloserine), D-CS-loaded alginate-chitosan nanoparticles, and physical mixture

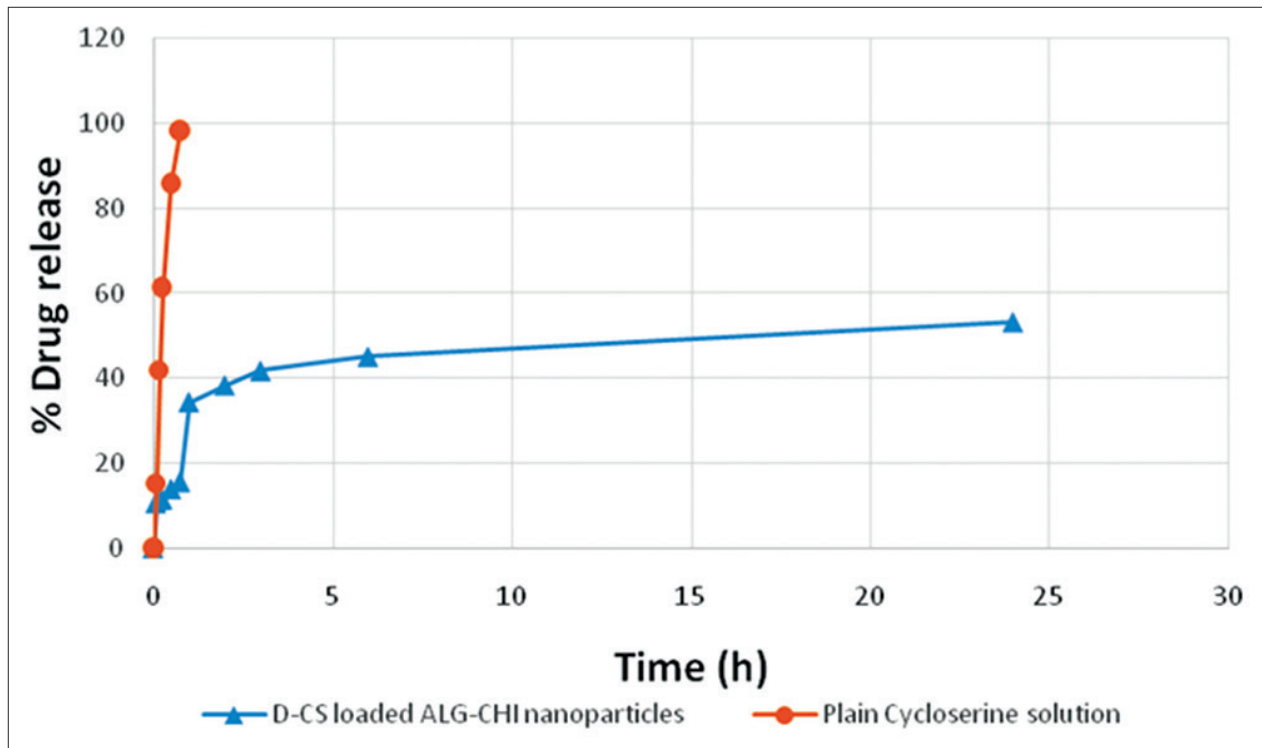

Fig. 12: In vitro release profile of D-cycloserine-loaded alginate-chitosan nanoparticles 
Table 10: The effect of storage condition on drug content and particle size of nanoparticles

\begin{tabular}{|c|c|c|c|c|c|c|}
\hline \multirow[t]{2}{*}{ Period } & \multicolumn{3}{|c|}{ Percentage of drug content } & \multicolumn{3}{|c|}{ Particle size (nm) } \\
\hline & $5^{\circ} \mathrm{C} \pm 3^{\circ} \mathrm{C}$ & $25^{\circ} \mathrm{C} \pm 2^{\circ} \mathrm{C}$ & $40^{\circ} \mathrm{C} \pm 2^{\circ} \mathrm{C}$ & $5^{\circ} \mathrm{C} \pm 3^{\circ} \mathrm{C}$ & $25^{\circ} \mathrm{C} \pm 2^{\circ} \mathrm{C}$ & $40^{\circ} \mathrm{C} \pm 2^{\circ} \mathrm{C}$ \\
\hline Initial & 100 & 100 & 100 & $344 \pm 5$ & $344 \pm 5$ & $344 \pm 5$ \\
\hline 1 month & $99.26 \pm 0.6$ & $97.79 \pm 0.3$ & $92.41 \pm 0.8$ & $346 \pm 4$ & $350 \pm 6$ & $392 \pm 7$ \\
\hline 2 months & $99.19 \pm 0.8$ & $95.43 \pm 0.6$ & $87.23 \pm 0.3$ & $347 \pm 2$ & $358 \pm 4$ & $404 \pm 2$ \\
\hline 3 months & $99.11 \pm 0.3$ & $92.92 \pm 0.6$ & $81.46 \pm 0.6$ & $348 \pm 6$ & $368 \pm 7$ & $413 \pm 4$ \\
\hline 6 months & $98.84 \pm 0.3$ & $80.52 \pm 0.3$ & $60.38 \pm 0.8$ & $388 \pm 6$ & $409 \pm 2$ & $452 \pm 5$ \\
\hline
\end{tabular}

Mean \pm SD, $n=3$. SD: Standard deviation

followed by controlled release up to $24 \mathrm{hrs}$. The existence of free drug or surface-adsorbed drug of the nanoparticles may give rise to initial burst release, while a controlled release was seen due to diffusion mechanism which causes the drug release from rigid polymeric chains of gelled biodegradable sodium ALG [42]. The CHI and ALG have a hydrophilic nature which helps in rapid hydration of nanoparticles following by penetration of release medium and the drug release, as this may confirm that the factors responsible for the drug release are solubilization of nanoparticles in the release medium. The nanosize helps in providing large surface area for dissolution and sink condition which helps in initial burst release of drugs in the release medium. The absence of such a significant dilution process upon inhalation into lungs suggests that this fast release should not occur in vivo. The drug entrapped into the inner core compartment stayed firmly inside the D-CS-loaded ALG-CHI nanoparticles showing a very slow release even at sink conditions with $46.65 \%$ of the initially incorporated drug still being associated with the D-CS-loaded ALG-CHI nanoparticles even after $24 \mathrm{~h}$. Evaluation of the release profiles of the pure drug showed that almost all the D-CS-loaded ALG-CHI nanoparticles were released immediately after start of the study, suggesting that the developed nanoparticles can be used as an important platform for sustained drug release.

\section{Particle aerodynamic study}

$\mathrm{RF}$ was the ratio of FPD to RD and was expressed in percentage. RF for the D-CS-loaded ALG-CHI nanoparticles was found to be $52.37 \pm 0.7 \%$. As per the obtained results, FPD and RD were found to be $129.89 \mu \mathrm{g}$ and $248 \mu \mathrm{g}$, respectively. This results showed that the D-CS-loaded ALG-CHI nanoparticles was suited for deep lung delivery.

\section{In vivo lung inflammatory response studies}

Lung inflammatory response study in the Wistar rats showed less inflammatory response, suggesting biosafety of these D-CS-loaded ALGCHI nanoparticles.

\section{Biochemical study}

At 4 hrs after exposure, the groups exposed to nanoparticles showed slightly increased levels of TP in the BALF compared to the controls. The level of TP was $0.22 \mathrm{~g} / \mathrm{dL}$ at $37^{\circ} \mathrm{C}$ at $4 \mathrm{hrs}$ after exposure to nanoparticles compared to the level of control which was found to be $0.20 \mathrm{~g} / \mathrm{dL}$. Similarly, the level of LDH in the BALF showed no significant increase as compared to the control group level. The level of LDH was $140.60 \mathrm{U} / \mathrm{L}$ at $37^{\circ} \mathrm{C}$, while in the control group, $\mathrm{LDH}$ level was found to be $120.30 \mathrm{U} / \mathrm{L}$ at $37^{\circ} \mathrm{C}$.

\section{Lung histopathological studies}

A qualitative analysis of inflammation after inhalation of the nanoparticulate formulation was done by the histological study. In lungs, the appearance of the alveoli and its lining, macrophages, interstitial capillary status, inflammatory cell infiltration (cell type and degree), fibrosis, and bronchial tree status were studied. In lungs, no inflammation or any notable changes were observed in the bronchial tree, alveoli, and blood vessel of the control group (Fig. 13a). In nanoparticle-treated animals, the majority of the lung tissue in the rats had a normal appearance. Sections showed normal histological appearance. The alveoli were composed of a single layer of squamous

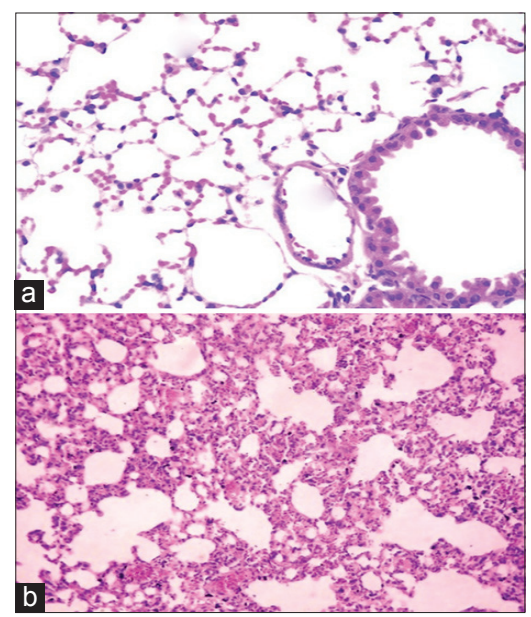

Fig. 13: Lung tissue histology. (a) $0.9 \%$ sodium chloride-treated lung tissue and, (b) D-cycloserine-loaded alginate-chitosan nanoparticles-treated lung tissue

epithelium. The interstitial connective tissue content was minimal. The vascular bed was rich and healthy. The bronchioles of various sizes were noted and were lined with columnar epithelium (larger bronchioles) and cuboidal epithelium (smaller bronchioles). Bronchiole associated lymphoid tissue (BALT) which are noted multifocally was within the normal histological limits (Fig. 13b). This suggested that the D-CSloaded ALG-CHI nanoparticles do not elicit a major inflammatory response and are therefore safe to be used in therapy.

\section{Stability studies}

The drug content and particle size of freeze-dried D-CS-loaded ALG-CHI nanoparticles were evaluated after the storage for 6 months at $5^{\circ} \mathrm{C} \pm 3^{\circ} \mathrm{C}$, $25^{\circ} \mathrm{C} \pm 2^{\circ} \mathrm{C}$, and $40^{\circ} \mathrm{C} \pm 2^{\circ} \mathrm{C}$, respectively. No aggregation was seen after the rehydration of nanoparticles stored at $5^{\circ} \mathrm{C} \pm 3^{\circ} \mathrm{C}$. No large deviation was seen in the drug content and particle size of nanoparticles at $5^{\circ} \mathrm{C} \pm 3^{\circ} \mathrm{C}$. However, the drug content was found to decrease and the size of the nanoparticles was found to be increased at $25^{\circ} \mathrm{C} \pm 2^{\circ} \mathrm{C}$ and $40^{\circ} \mathrm{C} \pm 2^{\circ} \mathrm{C}$, respectively. The physical stability of D-CS-loaded ALG-CHI nanoparticles can be stated from the results as shown in Table 10, which was obtained at $5^{\circ} \mathrm{C} \pm 3^{\circ} \mathrm{C}, 25^{\circ} \mathrm{C} \pm 2^{\circ} \mathrm{C}$, and $40^{\circ} \mathrm{C} \pm 2^{\circ} \mathrm{C}$, respectively. Thus, D-CS-loaded ALG-CHI nanoparticles exhibit a good storage stability at $5^{\circ} \mathrm{C} \pm 3^{\circ} \mathrm{C}$.

\section{CONCLUSION}

The controlled release formulations of D-CS-loaded ALG-CHI polyelectrolyte complex nanoparticles were successfully prepared by ionic gelation method. Concentration of drug, CHI, and sodium ALG were found to influence the particle size and entrapment efficiency of D-CS-loaded ALG-CHI nanoparticles. The $2^{3}$ factorial design revealed that the polymers and drug concentration significantly affected the response. The concentration of sodium ALG and CHI had a positive impact on entrapment efficiency and particle size, respectively, while drug concentration had a negative influence on the both entrapment 
efficiency and particle size. The application of factorial design gave a statistically systematic approach for the optimize formulation of D-CSloaded ALG-CHI nanoparticles with desired particle size and high entrapment efficiency with experimental validity. The SEM showed that the particles had a spherical shape and smooth surface. The FTIR, DSC, and XRD studies tend to show the ionic interaction between the polymers, thereby confirming the polyelectrolyte complex formation and also the entrapment of the drug into this polymeric matrix. The controlled release behavior of drug from optimized formulation can improve drug bioavailability and reduce dosing frequency which could be effective in overcoming patient noncompliance. In vitro deposition studies of D-CS-loaded ALG-CHI nanoparticles conducted by Twin stage impinger demonstrated a RF suitable for deep lung targeting. The formulation was also found to be well tolerated in rats. Taken together, these results indicate that these D-CS-loaded ALG-CHI nanoparticles could be a potential alternative to the existing conventional therapy in MDR-TB.

\section{ACKNOWLEDGMENT}

The authors thank Maneesh Pharmaceuticals (Mumbai), CIFT (Kochi), and Signet Chemicals (Mumbai) for gift samples of D-CS, CHI, and sodium ALG, respectively.

\section{REFERENCES}

1. Dye C, Watt CJ, Bleed DM, Hosseini SM, Raviglione MC Evolution of tuberculosis control and prospects for reducing tuberculosis incidence, prevalence, and deaths globally. JAMA 2005;293(22):2767-75

2. Gelperina S, Kisich K, Iseman MD, Heifets L. The potential advantages of nanoparticle drug delivery systems in chemotherapy of tuberculosis. Am J Respir Crit Care Med 2005;172(12):1487-90.

3. Faustini A, Hall AJ, Perucci CA. Risk factors for multidrug resistant tuberculosis in Europe: A systematic review. Thorax 2006;61(2):158-63.

4. Grosset JH, Singer TG, Bishai WR. New drugs for the treatment of tuberculosis: Hope and reality. Int $J$ Tuberc Lung Dis 2012;16(8):1005-14.

5. Li P, Dai YN, Zhang JP, Wang AQ, Wei Q. Chitosan-alginate nanoparticles as a novel drug delivery system for nifedipine. Int J Biomed Sci 2008;4(3):221-8

6. Justo OR, Moraes AM. Kanamycin incorporation in lipid vesicles prepared by ethanol injection designed for tuberculosis treatment. J Pharm Pharmacol 2005;57(1):23-30.

7. Irache JM, Merodio M, Arnedo A, Camapanero MA, Mirshahi M, Espuelas S. Albumin nanoparticles for the intravitreal delivery of anticytomegaloviral drugs. Mini Rev Med Chem 2005;5(3):293-305.

8. Umamaheshwari RB, Ramteke S, Jain NK. Anti-Helicobacter pylori effect of mucoadhesive nanoparticles bearing amoxicillin in experimental gerbils model. AAPS PharmSciTech 2004;5(2):e32.

9. Pandey R, Ahmad Z, Sharma S, Khuller GK. Nano-encapsulation of azole antifungals: Potential applications to improve oral drug delivery. Int J Pharm 2005;301(1-2):268-76.

10. Lucinda-Silva RM, Evangelista RC. Microspheres of alginate-chitosan containing isoniazid. J Microencapsul 2003;20(2):145-52.

11. Khuller GK, Kapur M, Sharma S. Liposome technology for drug delivery against mycobacterial infections. Curr Pharm Des 2004;10(26):3263-74.

12. Qurrat-ul-Ain, Sharma S, Khuller GK, Garg SK. Alginate-based oral drug delivery system for tuberculosis: Pharmacokinetics and therapeutic effects. J Antimicrob Chemother 2003;51(4):931-8.

13. Pandey R, Khuller GK. Chemotherapeutic potential of alginate-chitosan microspheres as anti-tubercular drug carriers. J Antimicrob Chemother 2004;53(4):635-40

14. Xiong MH, Bao Y, Yang XZ, Zhu YH, Wang J. Delivery of antibiotics with polymeric particles. Adv Drug Deliv Rev 2014;78:63-76.

15. Mi FL, Sung HW, Shyu SS. Drug release from chitosan- alginate complex beads reinforced by a naturally occurring crosslinking agent Carbohydr Polym 2002;48(1):61-72.

16. Coppi G, Iannuccelli V, Leo E, Bernabei MT, Cameroni R. Protein immobilization in crosslinked alginate microparticles. J Microencapsul 2002;19(1):37-44.

17. Kulkarni PV, Keshavayya J. Chitosan - Sodium alginate biodegradable Interpenetrating Polymer Network (IPN) beads for delivery of ofloxacin hydrochloride. Int J Pharm Pharm Sci 2010;2 Suppl 2:77-82.

18. Reddy BV, Kumar KH, Chandra SR, Chandra AS, Babu GD, Chandra P. Preparation and in-vitro evaluation of ofloxacin mucoadhesive microspheres. Int J Pharm Pharm Sci 2012;4(1):93-6.

19. Sathali AA, Varun J. Formulation, development and in vitro evaluation of candesartan cilexetil mucoadhesive microbeads. Int J Curr Pharm Res 2012;4(3):109-18.

20. V'Ooteghem MM. In: Edman P, editor. Biopharmaceutics of Ocular Drug Delivery. Boca Raton: CRC Press; 1993. p. 27-41.

21. Rajaonarivony M, Vauthier C, Couarraze G, Puisieux F, Couvreur P. Development of a new drug carrier made from alginate. J Pharm Sci 1993;82(9):912-7.

22. Arora S, Gupta S, Narang RK, Budhiraja RD. Amoxicillin loaded chitosan-alginate polyelectrolyte complex nanoparticles as mucopenetrating delivery system for $H$. Pylori. Sci Pharm 2011;79(3):673-94.

23. Motwani SK, Chopra S, Talegaonkar S, Kohli K, Ahmad FJ, Khar RK. Chitosan-sodium alginate nanoparticles as submicroscopic reservoirs for ocular delivery: Formulation, optimisation and in vitro characterisation. Eur J Pharm Biopharm 2008;68(3):513-25.

24. Mali AJ, Pawar AP, Purohit RN. Development of budesonide loaded biopolymer based dry powder inhaler: Optimization, in vitro deposition, and cytotoxicity study. J Pharm (Cairo) 2014;2014:795371.

25. Jabłczyńska K, Janczewska M, Kulikowska A, Sosnowski T. Preparation and characterization of biocompatible polymer particles as potential nanocarriers for inhalation therapy. Int J Polym Sci 2015;2015:1-8.

26. Kondej D, Sosnowski TR. Changes in the activity of the pulmonary surfactant after contact with bentonite nanoclay particles. Chem Eng Trans 2012;26:531-6.

27. Mann JF, McKay PF, Arokiasamy S, Patel RK, Klein K, Shattock RJ. Pulmonary delivery of DNA vaccine constructs using deacylated PEI elicits immune responses and protects against viral challenge infection. J Control Release 2013;170(3):452-9.

28. Sarmento B, Ferreira D, Veiga F, Ribeiro A. Characterization of insulinloaded alginate nanoparticles produced by ionotropic pre-gelation through DSC and FTIR studies. Carbohydr Polym 2006;66(1):1-7.

29. Malesu VK, Sahoo D, Nayak PL. Chitosan-sodium alginate nanocomposites blended with cloisite $30 \mathrm{~b}$ as a novel drug delivery system for anticancer drug curcumin. Int J Appl Biol Pharm Technol 2011;2(3):402-11

30. Emami J, Boushehri MS, Varshosaz J. Preparation, characterization and optimization of glipizide controlled release nanoparticles. Res Pharm Sci 2014;9(5):301-14

31. Desai TR, Hancock RE, Finlay WH. A facile method of delivery of liposomes by nebulization. J Control Release 2002;84(1-2):69-78.

32. Matilainen L, Järvinen K, Toropainen T, Näsi E, Auriola S, Järvinen T, et al. In vitro evaluation of the effect of cyclodextrin complexation on pulmonary deposition of a peptide, cyclosporin A. Int J Pharm 2006;318(1-2):41-8

33. Haberl N, Hirn S, Wenk A, Diendorf J, Epple M, Johnston BD, et al. Cytotoxic and proinflammatory effects of PVP-coated silver nanoparticles after intratracheal instillation in rats. Beilstein $J$ Nanotechnol 2013;4:933-40.

34. Neupane YR, Sabir MD, Ahmad N, Ali M, Kohli K. Lipid drug conjugate nanoparticle as a novel lipid nanocarrier for the oral delivery of decitabine: Ex vivo gut permeation studies. Nanotechnology 2013;24(41):415102.

35. Patel JK, Patel RP, Amin AF, Patel MM. Formulation and evaluation of mucoadhesive glipizide microspheres. AAPS PharmSciTech 2005;6(1):E49-55.

36. Gazori T, Khoshayand MR, Azizi E, Yazdizadeh PN, Nomani A, Haririan I. Evaluation of alginate/chitosan anoparticles as antisense delivery vector: Formulation, optimization and in vitro characterization. Carbohydr Polym 2009;77(3):599-606.

37. Sartori C, Finch D, Ralph B, Gilding K. Determination of the cation content of alginate thin films by FTIR spectroscopy. Polymer 1997;38(1):43-51.

38. Sankalia MG, Mashru RC, Sankalia JM, Sutariya VB. Reversed chitosan-alginate polyelectrolyte complex for stability improvement of alpha-amylase: Optimization and physicochemical characterization. Eur J Pharm Biopharm 2007;65(2):215-32.

39. Chen SC, Wu YC, Mi FL, Lin YH, Yu LC, Sung HW. A novel pHsensitive hydrogel composed of N,O-carboxymethyl chitosan and alginate cross-linked by genipin for protein drug delivery. J Control Release 2004;96(2):285-300.

40. Yoksan R, Jirawutthiwongchai J, Arpo K. Entrapment of ascorbyl 
palmitate in chitosan nanoparticles by oil-in-water emulsion and ionic gelation processes. Colloids Surf B Biointerfaces 2010;76(1):292-7.

41. Qi L, Xu Z, Jiang X, Hu C, Zou X. Preparation and antibacterial activity of chitosan nanoparticles. Carbohydr Res 2004;339(16):2693-700.
42. Zhang LF, Yang DJ, Chen HC, Sun R, Xu L, Xiong ZC, et al. An ionically crosslinked hydrogel containing vancomycin coating on a porous scaffold for drug delivery and cell culture. Int J Pharm 2008;353(1-2):74-87. 